\title{
Nonunitary geometric phases: A qubit coupled to an environment with random noise
}

\author{
Fernando C. Lombardo* and Paula I. Villar ${ }^{\dagger}$ \\ Departamento de Física Juan José Giambiagi, FCEyN UBA and IFIBA CONICET-UBA, Facultad de Ciencias Exactas y Naturales, \\ Ciudad Universitaria, Pabellón I, 1428 Buenos Aires, Argentina
}

(Received 26 December 2012; published 29 March 2013)

\begin{abstract}
We describe the decoherence process induced on a two-level quantum system in direct interaction with a nonequilibrium environment. The nonequilibrium feature is represented by a nonstationary random function corresponding to the fluctuating transition frequency between two quantum states coupled to the surroundings. In this framework, we compute the decoherence factors which have a characteristic "dip" related to the initial phases of the bath modes. We therefore study different types of environments, namely, ohmic and supraohmic. These environments present different decoherence time scales than the thermal environment we used to study. As a consequence, we compute analytically and numerically the nonunitary geometric phase for the qubit in a quasicyclic evolution under the presence of these particular nonequilibrium environments. We show in which cases decoherence effects could, in principle, be controlled in order to perform a measurement of the geometric phase using standard procedures.
\end{abstract}

DOI: 10.1103/PhysRevA.87.032338

PACS number(s): 03.67.Lx, 03.67.Pp, 05.40.Ca

\section{INTRODUCTION}

The spin-boson model is studied in a variety of fields, such as condensed matter physics, quantum optics, physical chemistry, and quantum information science [1] in order to describe nonunitary effects induced in quantum systems due to a coupling with an external environment. For a quantum system, the influence of the surroundings plays a role at a fundamental level. When the environment is taken into consideration, the system dynamics can no longer be described in terms of pure quantum states and unitary evolution. From a practical point of view, all real systems interact with an environment to a greater or lesser extent, which means that we expect their quantum evolution to be plagued by nonunitary effects, namely, dissipation and decoherence.

Most theoretical investigations of how the system is affected by the presence of an environment have been done using a thermal reservoir, usually assuming Markovian statistical properties and defined bath correlations [2,3] (there are also works on non-Markovian models as just, for example, Ref. [4]). However, there has been some growing interest in modeling more realistic environments, sometimes called "composite" environments [5-7]. In fact, the are many situations where the environment is better modeled by a nonequilibrium bath. Quantum dynamics in nonequilibrium environments has been previously considered by some recent investigations. For example, light-induced ultrafast coherent electronic processes in chemical or biological systems may occur on sufficiently short time scales [8]. In these cases, initial nonequilibrium states induced in the bath through the coupling among system and environment might not have the chance to reach equilibrium rapidly. Then, the transient nonequilibrium bath dynamics may undergo a nontrivial interaction with the system of interest in comparable time scales. Gordon et al. discussed the control of quantum coherence and the suppression of dephasing by stochastic control fields [9]. In

\footnotetext{
*lombardo@df.uba.ar

†paula@df.uba.ar
}

Ref. [5], the decoherence process induced by a nonequilibrium environment described by several equilibrium baths at different temperatures is discussed. Therein, it was suggested that the effect of such an environment on the quantum system could be described as the effect done by a single effective bath with a time-dependent temperature. The decoherence of single trapped ions coupled to engineered reservoirs, where the internal state and coupling can be controlled, was studied in Ref. [10].

In this context, we shall describe a simple model which gives a different insight into the behavior of a quantum system coupled to an environment that is not at thermal equilibrium. Herein, we study the dynamics of quantum coherence in nonequilibrium. We consider a two-level quantum system in a nonequilibrium bath, represented by random perturbations with nonstationary statistics. Therefore, we shall study how the quantum system is affected by the decoherence induced by the environment. We shall compare this decoherence process with the usual results for a thermal environment.

From another point of view, a system can retain the information of its motion when it undergoes a cyclic evolution in the form of a geometric phase (GP), which was first put forward by Pancharatman in optics [11] and later studied explicitly by Berry in a general quantal system [12]. Since then, great progress has been achieved in this field. As an important evolvement, the application of the geometric phase has been proposed in many fields, such as the geometric quantum computation. Due to its global properties, the geometric phase is propitious to construct fault-tolerant quantum gates. In this line of work, many physical systems have been investigated to realize geometric quantum computation, such as nuclear magnetic resonance (NMR) [13], Josephson junction [14], ion trap [15], and semiconductor quantum dots [16]. The quantum computation scheme for the GP has been proposed based on the Abelian or non-Abelian geometric concepts, and the GP has been shown to be robust against faults in the presence of some kind of external noise due to the geometric nature of the Berry phase [17-19]. It was therefore seen that the interactions play an important role for the realization of some specific operations. As the gates operate 
slowly compared to the dynamical time scale, they become vulnerable to open system effects and parameter fluctuations that may lead to a loss of coherence. Consequently, the study of the GP was soon extended to open quantum systems. Following this idea, many authors have analyzed the correction to the GP under the influence of an external thermal environment using different approaches (see Refs. [20-23] and references therein).

In this paper, we shall study how the GP is affected by the presence of a nonequilibrium environment. We shall consider a two-state quantum system coupled to such an environment and derive the corresponding decoherence factor in Sec. II. We shall analyze the decoherence process for ohmic and nonohmic environments. In Sec. III, we shall derive the GP for a nonunitary evolution of the quantum system in the presence of a nonequilibrium environment and compute how the GP is corrected in each case. Finally, in Sec. IV, we shall make our final remarks.

\section{PURELY DEPHASING SOLVABLE SPIN-BOSON MODEL}

A paradigmatic model of open quantum systems is a twostate quantum system coupled to a thermal environment. This is a particular case of the spin-boson model by Leggett [1] (where the tunneling bare matrix element is $\Delta=0$ ) and has been used by many authors to model decoherence in quantum computers [24] and, in particular, it is extremely relevant to the proposal for observing GPs in a superconducting nanocircuit [25]. In spite of its simplicity, this model captures many of the elements of decoherence theories and sheds some insight into the modification of the GPs due to the presence of the environment. The interaction between the two-state system and the environment is entirely represented by a Hamiltonian in which the coupling is only through $\sigma_{z}$. In this particular case, $\left[\sigma_{z}, H_{\mathrm{int}}\right]=0$ and the corresponding master equation for the reduced density matrix is much simplified, with no frequency renormalization and dissipation effects. In other words, the model describes a purely decohering (dephasing) mechanism, solely containing the diffusion term $\mathcal{D}(t)$ [21]. In such a case, it is easy to check that

$$
\rho_{\mathrm{r}_{01}}(t)=e^{-i \Omega t} e^{-\mathcal{A}(t)} \rho_{\mathrm{r}_{01}}(0)
$$

is the solution for the off-diagonal terms of the reduced density matrix (while populations remain constant) and $\mathcal{A}=$ $\int_{0}^{\infty} d s \mathcal{D}(s)$. $\Omega$ refers to the angular frequency of precession of a spin precessing the $z$ axis as ruled by the isolated from the environment Hamiltonian $H_{0}=\frac{1}{2} \Omega \sigma_{z}$ (responsible for the unitary evolution). The spin-boson model is the one used in Ref. [21] in order to present a solvable model to study how the GPs are corrected by decoherence in open systems. In that framework, we have studied not only how the GPs are corrected by the presence of the different type of environments but estimated the corresponding times at which decoherence become effective as well. These estimations should be taken into account when planning experimental setups, as the one performed in Ref. [26], where using a NMR quantum simulator, the geometric phase of an open system undergoing nonunitary evolution has been obtained. The GP was computed in a tomographic manner, measuring the off-diagonal elements of the reduced density matrix of the system. This study of the GP in the nonunitary regime is particularly important for the application of fault-tolerant quantum computation (see Ref. [27] as an example of measuring the Berry phase in a solidstate qubit where there is an important geometric contribution to dephasing that occurs when geometric operations are carried out in the presence of low-frequency noise).

In the present paper, we shall adopt a different model of decoherence than the one in Ref. [21]. We are concerned with nonequilibrium situations, in which the qubit (the main quantum system) is coupled to a nonequilibrium bath. The twolevel quantum system presents an energy gap $E_{2}(t)-E_{1}(t)=$ $\hbar \omega(t)$ which fluctuates due to the influence of the environment, where $E_{j}(t)$, with $j=1,2$ is the instantaneous energy of state $j$ as perturbed by the surroundings. Following the idea proposed in Ref. [28], the bath is represented by a random function of time corresponding to the transition frequency of the two-state quantum system. In contrast to the usual treatment, the statistical properties of this random function are nonstationary, corresponding physically to, for example, impulsively excited phonons of the environment with initial phases that are not random, but which have defined values at $t=0$. Due to this assumptions, this environment is not at thermal equilibrium. The time-dependent frequency is written in the form $\omega(t)=\Omega+\delta \omega(t)$, where $\delta \omega(t)$ is defined as

$$
\delta \omega(t)=\sum_{k=1}^{\infty} c_{k} \cos \left[\omega_{k} t+\theta_{k}(t)\right] .
$$

The Fourier components $c_{k}$ are positive constants related to the spectral density of the environment and the coupling of the bath modes to the system. It is important to mention that, in this model, the randomness enters through the nonstationary distribution of random phases $\theta_{k}(t)$, which are given by $\theta_{k}(t)=$ $\theta_{k}(0)+x_{k}(t)$. The random function $x_{k}(t)$ satisfies a diffusion equation

$$
\partial_{t} P_{k}(x, t)=D_{k} \partial_{x}^{2} P_{k}(x, t)
$$

where $P_{k}(x, t)$ is a time-dependent probability distribution and $D_{k}$ is the diffusion constant. The quantity $x_{k}$ is an angle, so $P(x, t)$ is a function with period $2 \pi$. The time-dependent probability distribution for component $k$ that solves Eq. (2) with an initial localized condition $P(x, 0)=\delta(x)$ is

$$
P_{k}(x, t)=\frac{1}{2 \pi}+\frac{1}{\pi} \sum_{n=1}^{\infty} e^{-n^{2} D_{k} t} \cos (n x) .
$$

This means that, physically, the phase of each component of the random force is not random at $t=0$, when an impulsive excitation creates a quantum coherence in the system, but decays to a uniform $1 / 2 \pi$ distribution under diffusive evolution with diffusion constant $D_{k}$ [28].

Following this approach, it is possible to evaluate

$$
\rho_{\mathrm{r}_{01}}(t)=e^{-i \Omega t}\left\langle e^{-i \int_{0}^{t} \delta \omega(s) d s}\right\rangle \rho_{\mathrm{r}_{01}}(0) \equiv e^{-i \Omega t} \mathcal{F}(t) \rho_{\mathrm{r}_{01}}(0),
$$

the solution for the off-diagonal element of the density matrix (while the populations remain constant again). Here, we denote with $\langle\cdots\rangle$ the nonequilibrium average over the nonstationary random bath and $\mathcal{F}(t)$ is defined as the decoherence factor.

By considering the typical factor $f_{k}(t)=$ $\exp \left[-i \int_{0}^{t} \delta \omega_{k}(s) d s\right]$, one has to do some algebra to 
obtain $|\mathcal{F}(t)|$. This mainly consists of performing the time integral and the averaging $f_{k}(t)$ over the distribution probability $P_{k}\left(x_{k}, t\right)$ [28]. After these computations, a simple but accurate approximation can be obtained, namely,

$$
|\mathcal{F}(t)|=\left|\prod_{k} f_{k}(t)\right| \simeq e^{-\beta(t)},
$$

with

$$
\begin{aligned}
\beta(t)= & \frac{1}{4} \int_{0}^{\infty} d \omega I(\omega)\left[1-e^{-2 D t}+\left(e^{-2 D t}-e^{-4 D t}\right)\right. \\
& \times \cos \{2[\omega t+\theta(\omega)]\}] .
\end{aligned}
$$

It is important to note that in Eq. (6), the continuum limit has already been taken (in the number of bath modes) and the diffusion constant has been assumed $D(\omega)=D$ for simplicity.

In order to study the decoherence process induced in the system by the presence of a nonequilibrium environment, we define a widely used physical spectral density $I(\omega)=$ $4 \gamma / \Lambda^{2} \omega^{n} / \Lambda^{n-1} e^{-\omega / \Lambda}[1]$, where $\gamma$ is the dimensionless dissipative constant and $\Lambda$ is the cutoff frequency. On general grounds, $\Lambda$ is the biggest frequency present in the environment, i.e., the frequency range of the environmental modes. In particular, the case with $n=1$ is the "ohmic" case and the one with $n>1$ is the "supraohmic" case. The ohmic environment is the most studied case in the literature, for example, in the quantum Brownian motion paradigm, and produces a dissipative force that in the limit of the frequency cutoff $\Lambda \rightarrow 0$ is proportional to the velocity. The supraohmic case, on the one hand, is generally used to model the interaction between defects and phonons in metals [1] and also to mimic the interaction between a charge and its own electromagnetic field (see, for example, Ref. [29]). In particular, the use of the supraohmic case can be used as a toy model to study decoherence process in quantum field theory [30].

This model of nonequilibrium is characterized by a key quantity which considers the effect of the initial phases of the bath modes in the function $\theta(\omega)$. In this case, we consider a linear dependence such as $\theta(\omega)=-\lambda \omega$. It is interesting to have the possibility to control dephasing by varying the single parameter $\lambda$. Following Eq. (6), the decoherence factors can be exactly calculated and they are given by

$$
\mathcal{F}_{\text {ohmic }}=e^{-\gamma e^{-4 D t}\left(-1+e^{2 D t}\right)\left[e^{2 D t}+\frac{1-4 \Lambda^{2}(t-\lambda)^{2}}{\left[1+4 \Lambda^{2}(t-\lambda)^{2}\right]^{2}}\right]}
$$

for the ohmic case, and

$$
\mathcal{F}_{\text {supra }}=e^{-6 \gamma e^{-4 D t}\left(-1+e^{2 D t}\right)\left[e^{2 D t}+\frac{1-24(t-\lambda)^{2} \Lambda^{2}+16(t-\lambda)^{4} \Lambda^{4}}{\left[1+4(t-\lambda)^{2} \Lambda^{2}\right]^{4}}\right]}
$$

for the supraohmic case (we use $n=3$ throughout this article).

It is interesting to analyze the asymptotic behavior of the function $\beta(t)$. Both types of nonequilibrium environments produce a linear time dependence for the very short time scale $D t, \Lambda t \ll 1$, which induces a decoherence factor of the form $\mathcal{F} \sim \exp [-a \gamma t]$ (where $a$ is a constant with proper units). This is similar to the decoherence factor calculated in Ref. [21] for the case of an ohmic finite-temperature environment (just assuming that $\gamma \sim \gamma_{0} K_{B} T$ ). In this limit, decoherence is always an efficient process, unless the dissipative constant $\gamma$ is very small. In the long time limit $D t, \Lambda t \gg 1$, both $\beta$ functions (the one for the ohmic, and the one corresponding to the

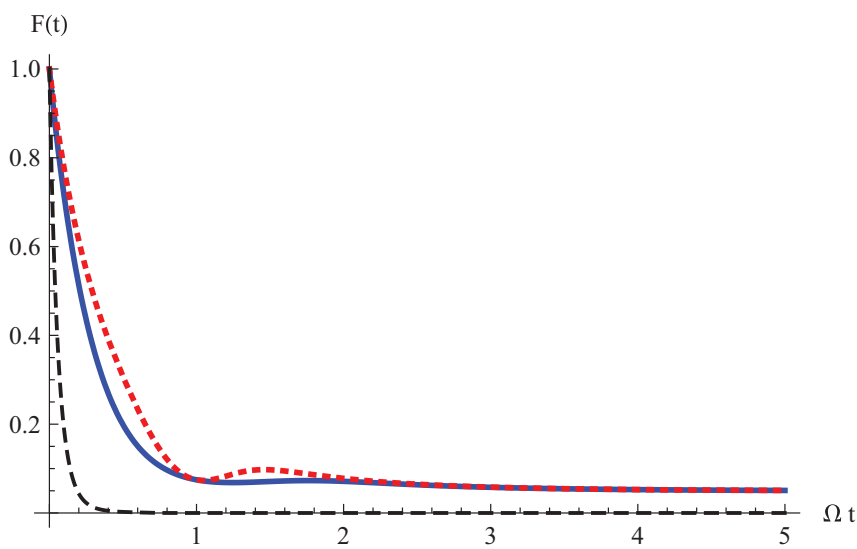

FIG. 1. (Color online) Evolution in time of the decoherence factor $\mathcal{F}(t)$ for different models of the environment in the strong coupling limit. The red short-dashed line is for an ohmic nonequilibrium bath and the long-dashed black line is for the supraohmic. For $D t$, $\Lambda t \gg 1$, the decoherence factor behaves as $\mathcal{F}_{\text {ohmic }}(t) \sim \exp (-\gamma)$ and $\mathcal{F}_{\text {supra }}(t) \sim \exp (-6 \gamma)$. We also present a solid blue line for the assumptions made in Ref. [8], a nonequilibrium bath with a Gaussian spectral density. Parameters used: $\gamma=3 ; \Lambda / \Omega=1$; $\Omega \lambda=1 ; D / \Omega=0.5$; and $n=3$ for the supraohmic environment.

supraohmic cases) acquire a constant value (different for each type of environment). In this long time regime, the decoherence factor in the ohmic case behaves as $\mathcal{F} \sim \exp [-\gamma]$, similar to the decoherence factor for the equilibrium supraohmic environment at zero temperature [21]. Meanwhile, in the supraohmic case, the decoherence factor approaches a long time value given by $\mathcal{F} \sim \exp [-6 \gamma]$. Again, as we mentioned before, in the case of small $\gamma$, decoherence never occurs, even at very long times. Intermediate times are ruled by the specific randomness introduced into the model. All in all, it is important to note the richness of the model which guarantees known and unknown decoherence processes by the correct tuning of the parameters.

In Fig. 1, we present the behavior of the decoherence factor for a strong dissipative case for both environments. As expected, the decoherence factor decays from unity to an asymptotic value. Therein, we also present the behavior of the decoherence factor found in Ref. [8], where a different spectral density to describe the environment has been used. The parameters used in the figure are similar to those used in Ref. [8] in order to do a better comparison and analysis. Unlike typical studies using the master equation in the weak-coupling limit $\left(\gamma \sim c_{k}^{2}\right)$, in the present approach there is no constraint for the value of $\gamma$. Therefore we can use either a strong or weak coupling as a value for $\gamma$. In the figure, we can note three different lines: the solid blue line for the results in Ref. [8], the red short-dashed line for our ohmic environment, and the solid black long-dashed line for the supraohmic environment. Then, it is easy to see that the ohmic case is very similar to the one obtained in Ref. [8], where a Gaussian spectral density was considered. In both cases, decoherence is very efficient, as expected since we are considering the overdamped case $(\gamma \geqslant 1)$. There is also an interesting fact: The supraohmic decoherence factor has a smaller decoherence time scale than the other two decoherence factors considered herein. This is 


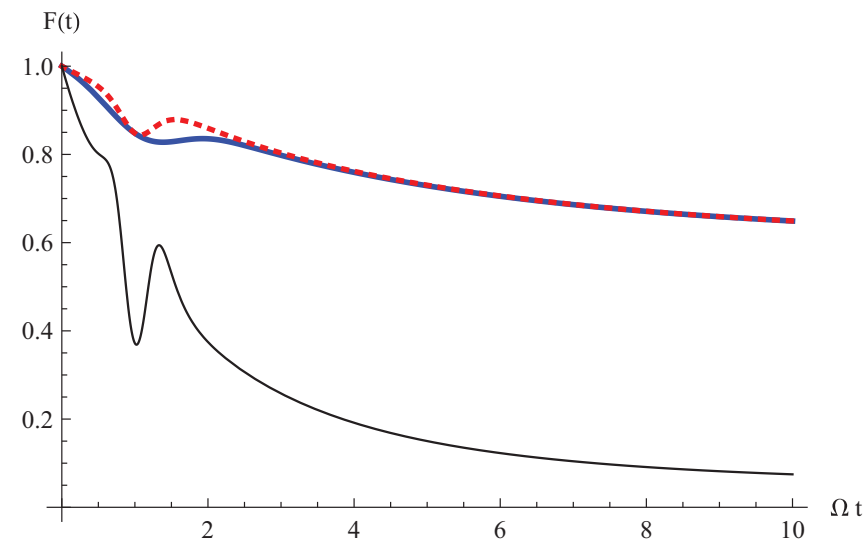

FIG. 2. (Color online) Evolution in time of the decoherence factor $F(t)$ for different models of the environment in the weak-coupling limit. The red short-dashed line is for an ohmic nonequilibrium bath and the black solid line (botton line) is for the supraohmic case. We also present a solid blue line for the assumptions made in Ref. [8], a nonequilibrium bath with a Gaussian spectral density (different with respect to the normally used in the theory of quantum open systems theory). Parameters used: $\gamma=0.5 ; \Lambda / \Omega=1 ; \Omega \lambda=1 ; D / \Omega=0.1$.

unlike the case for the equilibrium supraohmic environment, where decoherence is effective only at high temperature. This modeling of the environment gives a decoherence factor which drops from its initial value toward an asymptotic value $[\mathcal{F}(t \rightarrow$ $\infty)$ ] after the intermediate time $t=\lambda$. At this time the system rephases back to the slowly decaying envelope that is not purely exponential. As $\lambda$ becomes large and positive, the decay approaches the envelope function without the nonmonotonic dip (that occurs at $t=\lambda$ ). Nonexponential behavior in the decay of quantum coherence has been observed in full manyparticle simulations of quantum coherent dynamics under nonequilibrium conditions [31].

In order to have a better view of the dip where the system "recoheres" for a while, we present the behavior of the decoherence factors for the weak-coupling case in Fig. 2. Therein, $\gamma$ has a smaller value, comparable to those we used when dealing with environments in thermal equilibrium in the underdamped case $[21,32,33]$. The dip is obtained by introducing, as we mentioned before, the simple relation $\theta(\omega)=-\lambda \omega$ for the initial phases of the bath modes in the modeling of the environment. Even though this assumption is a minimalistic model, it allows to have some kind of control in the decoherence process which in turn can be useful in experimental setups where decoherence is always an obstacle to overcome. This result agrees with the one in Ref. [5], where it was shown that nonequilibrium decoherence can be slowed down in a controlled manner as compared to the corresponding equilibrium situation.

A different modeling of the initial phases of the bath modes can, in principle, be adopted. However, herein we use the linear one just for simplicity. A complex assumption can be, for example, $\theta(\omega)=-\lambda \omega^{2}$. The derivation of the decoherence factor is somewhat more difficult and is not worth writing explicitly here. Anyway, the decoherence factor for a quadratic behavior in $\omega$ is presented in Fig. 3 for an ohmic and supraohmic nonequilibrium environment. In such a case, it is

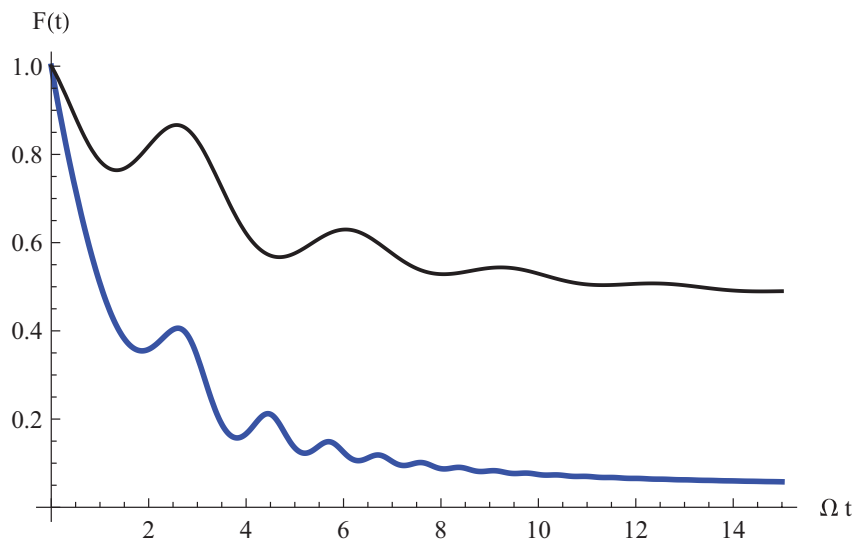

FIG. 3. (Color online) A more complex modeling of the initial phases of the bath modes by considering $\theta(\omega)=-\lambda \omega^{2}$ in an ohmic (blue solid line on the botton) and supraohmic (black solid line on top) environment. Parameters used: $\gamma=3 ; \Lambda / \Omega=1 ; \Omega \lambda=1 ; D / \Omega=$ 0.1 .

important to note a more complicated structure of dips in the decoherence factor.

\section{APPLICATION: GEOMETRIC PHASE FOR A QUBIT COUPLED TO A NONEQUILIBRIUM ENVIRONMENT}

In order to compute the GP and note how it is corrected by the environment, we shall briefly review the way the geometric phase can be computed for a system under the influence of external conditions such as an external bath. In Ref. [20], a quantum kinematic approach was proposed and the geometric phase (GP) for a mixed state under nonunitary evolution has been defined as

$$
\phi_{G}=\arg \left\{\sum_{k} \sqrt{\varepsilon_{k}(0) \varepsilon_{k}(\tau)}\left\langle\Psi_{k}(0) \mid \Psi_{k}(\tau)\right\rangle e^{-\int_{0}^{\tau} d t\left\langle\Psi_{k}\left|\frac{\partial}{\partial t}\right| \Psi_{k}\right\rangle}\right\},
$$

where $\varepsilon_{k}(t)$ are the eigenvalues and $\left|\Psi_{k}\right\rangle$ the eigenstates of the reduced density matrix $\rho_{\mathrm{r}}$ (obtained after tracing over the reservoir degrees of freedom). In the last definition, $\tau$ denotes a time after the total system completes a cyclic evolution when it is isolated from the environment. Taking into account the effect of the environment, the system no longer undergoes a cyclic evolution. However, we shall consider a quasicyclic path $\mathcal{P}: t \epsilon[0, \tau]$, with $\tau=2 \pi / \Omega(\Omega$ is the system's characteristic frequency). When the system is open, the original GP, i.e., the one that would have been obtained if the system had been closed $\phi_{U}$, is modified. This means, in a general case, that the phase can be interpreted as $\phi_{G}=\phi_{U}+\delta \phi_{G}$, where $\delta \phi_{G}$ depends on the kind of environment coupled to the main system [7,21-23,26,34].

Assuming an initial quantum state of the form

$$
|\psi(0)\rangle=\cos \left(\frac{\theta_{0}}{2}\right)|0\rangle+\sin \left(\frac{\theta_{0}}{2}\right)|1\rangle,
$$

its evolution at a later time $t$ is

$$
|\psi(t)\rangle=e^{-i \Omega t} \cos \left(\theta_{+}\right)|0\rangle+\sin \left(\theta_{+}\right)|1\rangle
$$




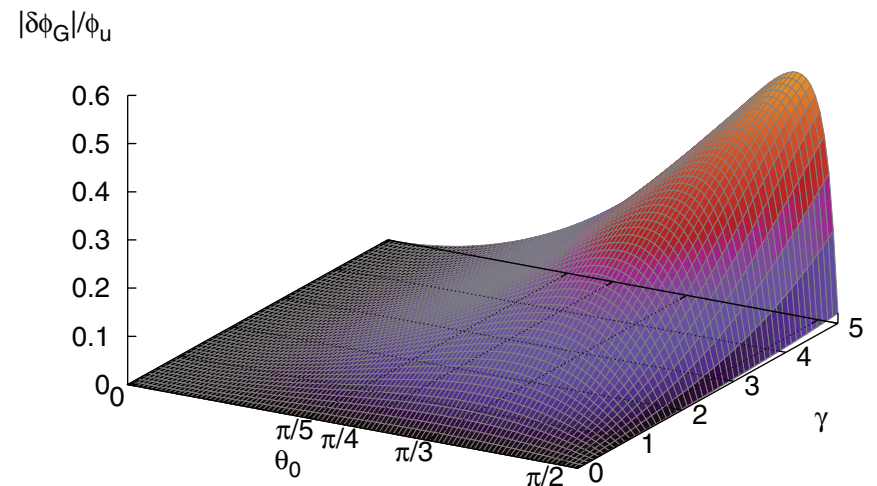

FIG. 4. (Color online) Behavior of the geometric phase as a function of the initial state of the quantum system $\theta_{0}$ (measured in radians) and the dissipation of the environment (dimensionless $\gamma$ ) induced by an ohmic nonequilibrium environment in a cycle. Parameters used: $\Lambda / \Omega=1 ; \Omega \lambda=1 ; D / \Omega=0.1$.

where

$$
\begin{aligned}
& \cos \left(\theta_{+}\right)=\frac{\sin \left(\theta_{0}\right)|\mathcal{F}(t)|}{\sqrt{\sin ^{2}\left(\theta_{0}\right)|\mathcal{F}(t)|^{2}+4\left[\varepsilon_{+}-\cos ^{2}\left(\frac{\theta_{0}}{2}\right)\right]^{2}}}, \\
& \sin \left(\theta_{+}\right)=\frac{2\left[\varepsilon_{+}-\cos ^{2}\left(\frac{\theta_{0}}{2}\right)\right]}{\sqrt{\sin ^{2}\left(\theta_{0}\right)|\mathcal{F}(t)|^{2}+4\left[\varepsilon_{+}-\cos ^{2}\left(\frac{\theta_{0}}{2}\right)\right]^{2}}},
\end{aligned}
$$

and $\varepsilon_{+}$the eigenvalue of the reduced density matrix, namely,

$$
\varepsilon_{+}=\frac{1}{2}\left[1+\sqrt{\cos ^{2}\left(\theta_{0}\right)+\sin ^{2}\left(\theta_{0}\right)|\mathcal{F}(t)|^{2}}\right],
$$

while $\varepsilon_{-}$does not contribute to the geometric case since $\varepsilon_{-}(t=$ $0)=0$ [see definition Eq. (9)].

As in our previous works $[7,21,26,34]$, the GP is obtained by computing eigenvectors and eigenvalues of the reduced density matrix and using Eq. (9),

$$
\phi_{G}=\Omega \int_{0}^{\tau} \cos ^{2}\left(\theta_{+}\right) d t .
$$

In Figs. 4 and 5 we plot the environmentally induced correction to the unitary phase $\left|\delta \phi_{G}\right|$ (normalized by the value of $\left.\phi_{U}\right)$ as a function of the system's initial quantum state

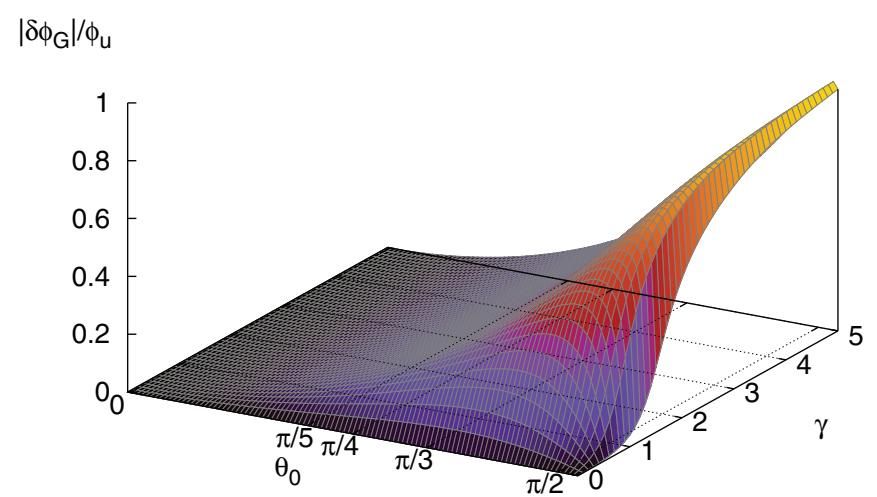

FIG. 5. (Color online) Behavior of the GP as a function of the initial state $\left(\theta_{0}\right.$ in radians) of the quantum system and the dissipation of the environment (dimensionless $\gamma$ ) induced by a supraohmic nonequilibrium environment in a cycle. Parameters used: $\Lambda / \Omega=1$; $\Omega \lambda=1 ; D / \Omega=0.1$. $\left(\theta_{0}\right)$ and the dissipation induced in the quantum subsystem due to the presence of the random environment $(\gamma)$. In both figures we have considered a wide range of values for $\gamma$, considering both weak and strong coupling between system and environment. For small values of $\gamma$, the GP behaves very similarly to the unitary GP, which is $\phi_{U}=\pi\left(1+\cos \theta_{0}\right)$. However, as we increase the value of $\gamma$, there is a notable change in the curvature as a function of $\theta_{0}$, leading to more values of $\theta_{0}$ with a null GP. As expected, the more decohering environment, the less survival of the GP. In agreement with Fig. 2, we can see that the geometric phase is less corrected (with respect to the isolated case) in the presence of an ohmic nonequilibrium environment rather than of a supraohmic one. This can be noted by the fact that Fig. 4 remains a smooth function of $\theta_{0}$ for bigger values of $\gamma$ than Fig. 5, in which case the phase rapidly behaves different as a function of the dissipation constant.

The GP cannot be fully computed analytically but we can perform an expansion in powers of the coupling constant to obtain an accurate approximation of it [21-23]. Hence, we expand in powers of $\gamma$ the $\cos ^{2} \theta_{+}$in Eq. (15), using the definition of the decoherence factors for each environment, namely, Eqs. (7) and (8). As mentioned before, the correction to the GP is defined as $\delta \phi_{G}$, while $\phi_{U}$ is the unitary GP. In the case of the ohmic nonequilibrium environment, the correction to the unitary GP is given by

$\delta \phi_{G_{n=1}} \approx \pi \gamma \sin ^{2}\left(\theta_{0}\right) \cos \left(\theta_{0}\right)+\gamma \frac{\Omega D}{\Lambda^{2}} e^{-2 D \lambda} \sin ^{2}\left(\theta_{0}\right) \cos \left(\theta_{0}\right)$.

Proceeding the same way for the supraohmic environment, the correction to the unitary GP is

$$
\begin{aligned}
\delta \phi_{G_{n=3}} \approx & 6 \pi \gamma \sin ^{2}\left(\theta_{0}\right) \cos \left(\theta_{0}\right) \\
& +\gamma \frac{\Omega D^{3}}{4 \Lambda^{4}} e^{-2 D \lambda} \sin ^{2}\left(\theta_{0}\right) \cos \left(\theta_{0}\right) .
\end{aligned}
$$

The corrections of the GP for both environments agree for small values of $\gamma$ in Figs. 4 and 5. In both cases, the dependence with the parameter $\lambda$ is exponentially negligible. Another interesting feature of the corrections of the GP is that they depend on the initial angle of the quantum state, and this dependence is in agreement with the ones obtained for a two-level system in interaction with environments at equilibrium [7,21-23,26,34]. Neglecting the small correction induced by $\lambda$ (which is a correct assumption seen Figs. 4 and 5), both cases are similar to the very low-temperature corrections found in Ref. [21] for the case of thermal environments. In Figs. 6 and 7, we show the range of validity of the first-order perturbative expansion in powers of $\gamma$. In Fig. 6, it is clear that the perturbative result (solid line) of Eq. (16) is in excellent agreement with the exact result (dashed line), even for not too small values of the coupling strength parameter $\gamma$. Figure 7 shows that Eq. (17) is also a good approximation to the exact result (dashed line), but only for very small values of $\gamma$.

It is an interesting feature to study which is the influence of the observed dip (in the decoherence factor) in the behavior of the GP. In this model of nonequilibrium environment, the parameter $\lambda$, which enters through the random phases, sets the position at which the "dip" or "recoherence" takes place. As we 


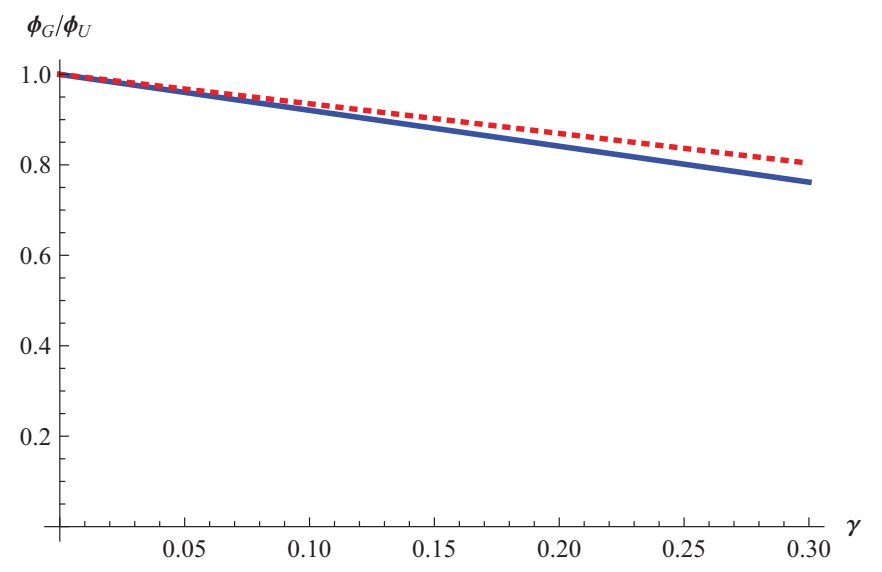

FIG. 6. (Color online) Comparison between exact GP (red dashed line), in the presence of an ohmic environment, and the first-order perturbative correction (blue solid line) from Eq. (16). Perturbative result is in good agreement with the exact result for a long range of values of $\gamma$. Parameters used: $D / \Omega=1, \Omega \lambda=1, \Lambda / \Omega=1$.

have seen, there is a time scale when the system seems to gain coherence $(t \sim \lambda)$. In Fig. 8, we present the correction to the $\mathrm{GP}\left|\delta \phi_{G}\right|$ as a function of the initial state of the quantum system $\left(\theta_{0}\right)$ and the initial phases of the bath modes $(\Omega \lambda)$ for an ohmic nonequilibrium environment. Therein, we can observe that the geometric phase is not affected by the dip in the decoherence factor, as it is computed over a quasicyclic evolution. In Fig. 8 we can note that the GP has a monotonous behavior as a function of $\lambda$. The analytical estimation of the influence of $\lambda$ in the correction to the GP, made in Eqs. (16) and (17), is also checked in Fig. 8.

\section{FINAL REMARKS}

The geometric phase of quantum states is an issue worthy of attention. It could be a potential application in holonomic quantum computation since the study of spin systems effectively allows us to contemplate the design of a solid-state quantum computer. However, decoherence is the main obstacle to overcome. All realistic quantum systems are coupled to

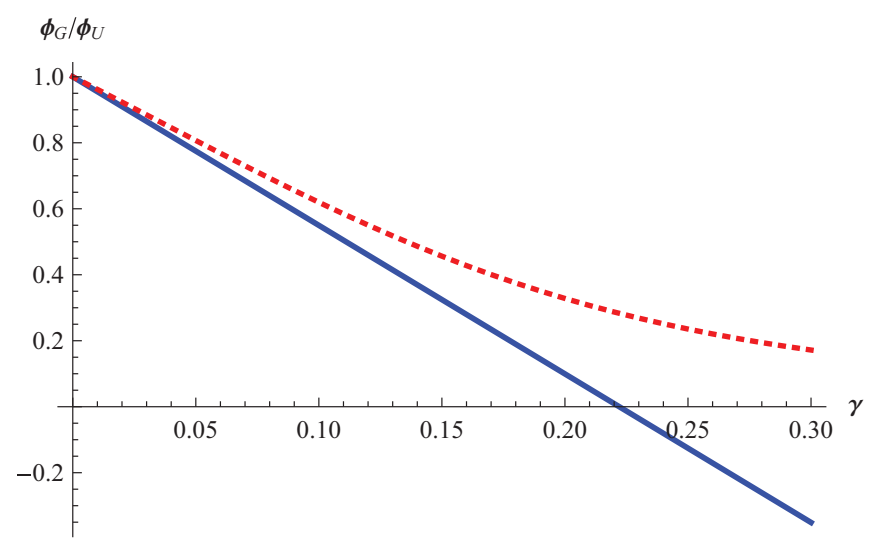

FIG. 7. (Color online) Comparison between exact geometric phase (red dashed line), in the presence of an supraohmic environment, and the first-order perturbative correction (blue solid line) from Eq. (17). The perturbative result is a good approximation for really small values of $\gamma$. Parameters used: $D / \Omega=1 ; \Omega \lambda=1 ; \Lambda / \Omega=1$.

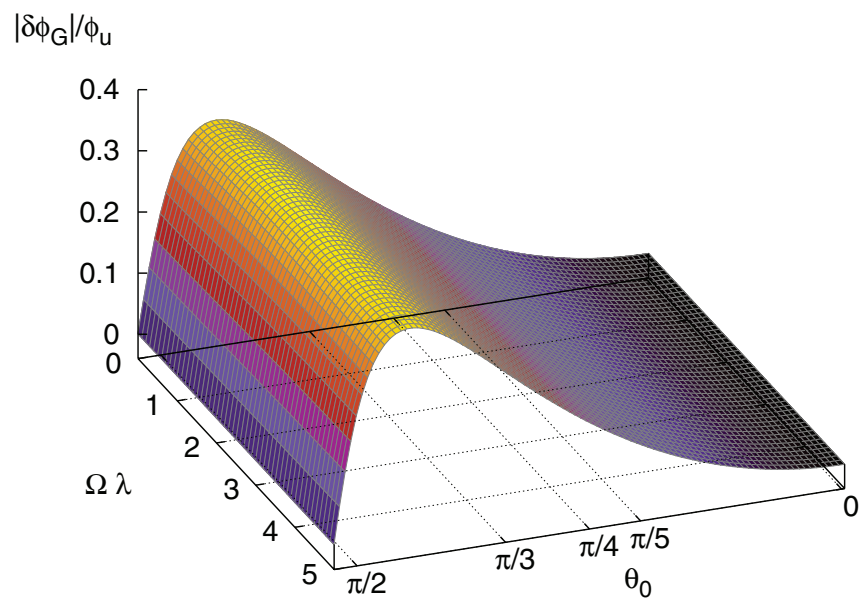

FIG. 8. (Color online) Behavior of the geometric phase as a function of the initial state $\theta_{0}$ (in radians) of the quantum system and the initial phases of the bath modes $(\lambda)$ in a cycle for an ohmic nonequilibrium environment. Parameters used: $\Lambda / \Omega=1 ; \gamma=3$; $D / \Omega=0.1$.

their surroundings to a greater or lesser extent. Furthermore, in most cases of practical interest, quantum systems are subjected to many noise sources with different amplitudes and correlation times, corresponding de facto to a nonequilibrium environment.

Herein, we have presented a simple case to illustrate the general phenomenon of dephasing in a nonequilibrium bath. We have studied the decoherence process of a quantum system in interaction with an initially nonequilibrium bath that can be controlled by manipulating the nature of the relative initial phases of the bath modes. The decoherence factors computed here suggest that by engineering these initial phases, the dephasing of the subsequent quantum evolution can potentially be controlled. We have found similarities and differences in the decoherence process between the environment presented here and thermal environments studied in previous works. The model presented here is another proposal for engineering reservoirs in a manner reminiscent of a coherent control experiment using shaped pulses [35]. In this model, the control parameter $\lambda$ is derived not from a laser pulse, but rather from well-defined phase relations between the modes of the bath. Another possible candidate for realizing this decoherence environment is to use the artificially generated fluctuating environments with NMR. It could be possible, in principle, to use the quantum simulator of Ref. [26] to generate the fluctuating phase $\theta(\omega)$ of the present proposal.

The analysis of the effect produced by decoherence on the GP is crucial at the time to design an experimental setup to measure the GP using, for example, interferometry. We found that the convenient nonequilibrium environment to observe GPs is the weak-coupled ohmic case. It is important that there is no restriction about zero-temperature environments in this case, as it was found in Ref. [21], as the most convenient scenario. In this framework, these kind of environments could become a proper experimental setup for the observation of the geometric phase. Therefore, we have computed the geometric phase for an ohmic and supraohmic nonequilibrium environment and have seen how they deviate from the unitary 
geometric phase. So far, we have seen that the characteristic dip of the decoherence factor does not affect much the geometric phase and that the ohmic nonequilibrium environments are not as destructive as the supraohmic nonequilibrium ones or the thermal environments we are so used to see in the literature. The effect done on the geometric phase by the ohmic (or/and supraohmic) nonequilibrium environment can be seen as similar to the one done by a single reservoir with an effective temperature, as the nonequilibrium environment model proposed in Ref. [5]. In the very weak-coupling limit, we have evaluated the corrections induced by the nonequilibrium environment on the unitary GP, showing that there is a small (exponentially suppressed) correction due to the random parameter $\lambda$. More general models should be analyzed in a future work.

\section{ACKNOWLEDGMENT}

This work is supported by CONICET, UBA, and ANPCyT, Argentina.
[1] A. J. Leggett, S. Chakravarty, T. A. Dorsey, M. P. A. Fisher, A. Garg, and W. Zwerger, Rev. Mod. Phys. 59, 1 (1987).

[2] J. P. Paz and W. H. Zurek, in Coherent Matter Waves, edited by R. Kaiser, C. Westbrook, and F. David, Proceedings of the Les Houches Summer School on Theoretical Physics, LXXII, 2000 (EDP Sciences/Springer, Berlin, 2001), pp. 533-614; W. H. Zurek, Rev. Mod. Phys. 75, 715 (2003).

[3] U. Weiss, Quantum Dissipative Systems (World Scientific, Singapore, 1999).

[4] H.-S. Goan, C.-C. Jian, and P.-W. Chen, Phys. Rev. A 82, 012111 (2010).

[5] J. Beer and E. Lutz, arXiv:1004.3921v2 [quant-ph].

[6] F. C. Lombardo and P. I. Villar, Phys. Rev. A 72, 034103 (2005).

[7] P. I. Villar and F. C. Lombardo, Phys. Rev. A 83, 052121 (2011).

[8] C. C. Martens, J. Phys. B 45, 154008 (2012).

[9] G. Gordon, G. Kurizki, S. Mancini, D. Vitali, and P. Tombesi, J. Phys. B 40, S61 (2007).

[10] C. J. Myatt et al., Nature (London) 403, 269 (2000).

[11] S. Pancharatnam, Proc. Indian Acad. Sci. A 44, 247 (1956).

[12] M. V. Berry, Proc. R. Soc. London A 392, 45 (1984).

[13] J. A. Jones, V. Vedral, A. Ekert, and G. Castagnoli, Nature (London) 403, 869 (2000).

[14] L. Faoro, J. Siewert, and R. Fazio, Phys. Rev. Lett. 90, 028301 (2003).

[15] J. E. Sonier, Science 292, 1695 (2001).

[16] P. Solinas, P. Zanardi, N. Zanghi, and F. Rossi, Phys. Rev. B 67, 121307 (2003).

[17] P. Zanardi and M. Rasetti, Phys. Lett. A 264, 94 (1999).

[18] Wang Xiang-Bin and M. Keiji, Phys. Rev. Lett. 87, 097901 (2001).
[19] E. Sjoqvist et al., New J. Phys. 14, 103035 (2012).

[20] D. M. Tong, E. Sjoqvist, L. C. Kwek, and C. H. Oh, Phys. Rev. Lett. 93, 080405 (2004); 95, 249902(E) (2005).

[21] F. C. Lombardo and P. I. Villar, Phys. Rev. A 74, 042311 (2006).

[22] F. C. Lombardo and P. I. Villar, Int. J. Quantum Inf. 6, 707713 (2008).

[23] P. I. Villar, Phys. Lett. A 373, 206 (2009).

[24] G. M. Palma, K. Suominen, and A. Ekert, Proc. R. Soc. London A 452, 567 (1996); L. Viola and S. Lloyd, Phys. Rev. A 58, 2733 (1998).

[25] G. Falci, R. Fazio, G. M. Palma, J. Siewert, and V. Vedral, Nature (London) 407, 355 (2000).

[26] F. M. Cucchietti, J.-F. Zhang, F. C. Lombardo, P. I. Villar, and R. Laflamme, Phys. Rev. Lett. 105, 240406 (2010).

[27] P. J. Leek, J. M. Fink, A. Blais, R. Bianchetti, M. Goppl, J. M. Gambetta, D. I. Schuster, L. Frunzio, R. J. Schoelkopf, and A. Wallraff, Science 318, 1889 (2007).

[28] C. C. Martens, J. Chem. Phys. 133, 241101 (2010).

[29] P. Sonnentag and F. Hasselbach, Phys. Rev. Lett. 98, 200402 (2007).

[30] F. Lombardo and F. D. Mazzitelli, Phys. Rev. D 53, 2001 (1996).

[31] J. M. Riga and C. C. Martens, J. Chem. Phys. 120, 6863 (2004); 322, 108 (2006).

[32] F. C. Lombardo and P. I. Villar, Phys. Lett. A 336, 16 (2005).

[33] F. C. Lombardo and P. I. Villar, Phys. Lett. A 371, 190 (2007).

[34] F. C. Lombardo and P. I. Villar, Phys. Rev. A 81, 022115 (2010).

[35] M. P. A. Branderhorst, P. Londero, P. Wasylczyk, C. Brif, R. L. Kosut, H. Rabitz, and I. A. Walmsley, Science 320, 638 (2008). 